DESY 99-152

September 1999

\title{
String breaking and lines of constant physics in the SU(2) Higgs model*
}

\author{
F. Knechtli \\ DESY, Platanenallee 6, D-15738 Zeuthen, Germany
}

\begin{abstract}
We present results for the ground state and first excited state static potentials in the confinement "phase" of the SU(2) Higgs model. String breaking and the crossing of the energy levels are clearly visible. We address the question of the cut-off effects in our results and observe a remarkable scaling of the static potentials.
\end{abstract}

\section{Introduction}

String breaking, the flattening of the static potential, has been observed by computing on the lattice the static (fundamental) potential in the three- and four-dimensional SU(2) Higgs model and the static adjoint potential in the threedimensional SU(2) Yang-Mills theory. In QCD, recent attempts with two flavors of dynamical quarks failed to observe string breaking 11. The problems in the extraction of the static potential in QCD arise from the poor overlap of the "string states", described by Wilson loops, with the ground state at large separations of the static quarks. The latter is better described in terms of two static-light mesons, which are bound states of a static quark and the light dynamical quark field. To overcome this problem one has to extract the static potential from a matrix correlation function in which "string" and "two-meson states" enter. This basic point was already noted in 2 .

We compute the potential between static charges in the fundamental representation of the gauge group (static quarks) in the fourdimensional SU(2) Higgs model in the confinement "phase", where it resembles QCD. We improve our first study [3] with a better lattice resolution and also determine the first excited state static potential. Furthermore, we address the question of finding lines of constant physics in order to study the scaling behavior. For a detailed exposition of our results, we refer to [ [ [].

\footnotetext{
*Talk given at 17th International Symposium on Lattice Field Theory (LATTICE 99), Pisa, Italy, June 29 - July 3, 1999 .
}

\section{String breaking}

We construct a matrix correlation function $C_{i j}(r, t)$ [3] using a basis of states $|i\rangle$ that contains string-type states, described by (smeared) Wilson lines, and meson-type states, described by (smeared) Higgs fields. The static potentials $V_{\alpha}(r)(\alpha=0,1,2, \ldots)$ are extracted from $C_{i j}(r, t)$ using the variational method proposed in [5]. Because of divergent (like $\frac{1}{a}$ ) self-energy contributions of the static charges, the static potentials $V_{\alpha}(r)$ need to be renormalised. This can be done by considering

$r_{0}\left[V_{\alpha}(r)-2 \mu\right]$.

The energy $\mu$ is the mass of a static-light meson, bound state of a static charge and the dynamical Higgs field. The multiplication with the reference scale $r_{0}$ [6] makes the physical quantity dimensionless. In Fig. A (circles), we show the ground state and first excited state static potentials (1) as functions of the distance $r / r_{0}$ for the bare parameter set $\beta=2.4, \kappa=0.2759$ and $\lambda=0.7$ (we adopt the conventional notation of $[7]$ ). The ground state potential shows an approximate linear rise at small distances: around distance

$r_{\mathrm{b}} \approx 1.9 r_{0}$

the potential flattens. The string breaks. As expected, for large distances the potential approaches the asymptotic value $2 \mu$. The first excited potential comes very close to the ground state potential around $r_{\mathrm{b}}$ and rises linearly at larger distances.

We would like to get an insight into the interplay between string-type and meson-type states. We consider the diagonal sub-blocks of the matrix correlation function corresponding to string- 


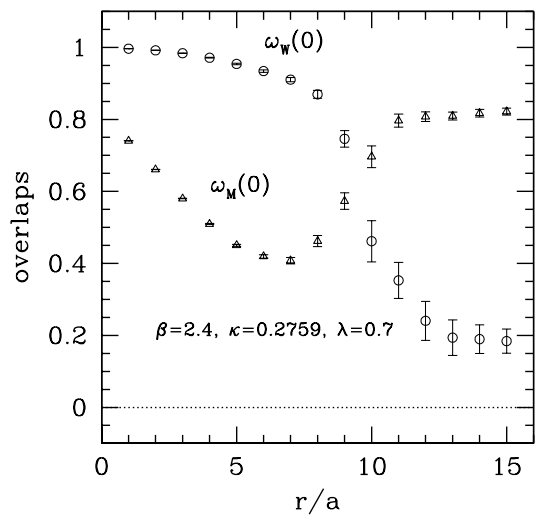

Figure 1. Here, we show the overlaps of the stringtype (circles) and meson-type (triangles) states with the ground state of the Hamiltonian.

type and to meson-type states separately and determine approximate ground state wave functions $v_{0}^{\mathrm{W}}$ for the string-type states and $v_{0}^{\mathrm{M}}$ for the meson-type states. We then construct a projected matrix correlation function

$$
\begin{aligned}
\Omega_{k l}(t) & =v_{0, i}^{k} C_{i j}(t) v_{0, j}^{l} \quad(k, l=\mathrm{W}, \mathrm{M}) \\
& =\sum_{\alpha} \omega_{l}(\alpha) \omega_{k}(\alpha) \mathrm{e}^{-t V_{\alpha}(r)}
\end{aligned}
$$

where $\alpha$ labels the true eigenstates of the Hamiltonian and the real coefficients $\omega_{k}(\alpha)$ are the overlaps of the string-type $(k=\mathrm{W})$ and meson-type $(k=\mathrm{M})$ states with the eigenstates of the Hamiltonian. The overlaps with the ground state are shown in Fig. 1 and with the first excited eigenstate in Fig. 22 (we choose the sign conventions $\omega_{\mathrm{W}}(0)>0$ and $\left.\omega_{\mathrm{W}}(1)>0\right)$. In the string breaking region around $r / a=9-10$, the overlaps of the string-type and meson-type states have similar magnitude, both in Fig. 1 and Fig. 2. The scenario of string breaking as a level crossing phenomenon [8] is confirmed. We would like to point out that the overlaps represented in Fig. 1 and Fig. 2 are not quantities which have a continuum limit.

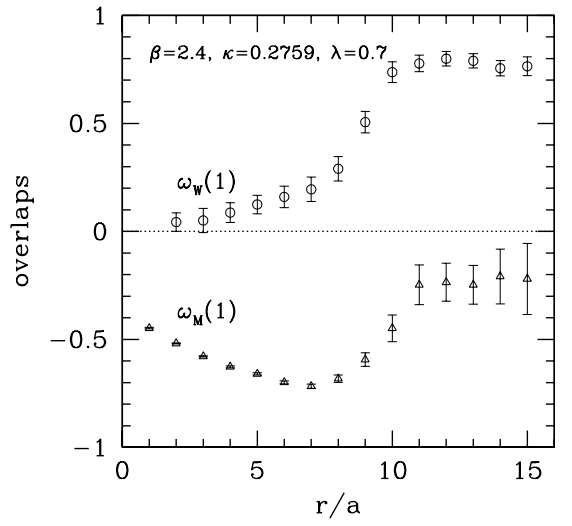

Figure 2. Here, we show the overlaps of the stringtype (circles) and meson-type (triangles) states with the first excited eigenstate of the Hamiltonian.

\section{Lines of constant physics}

In order to investigate the presence of lattice artifacts in dimensionless physical quantities such as (11), we must vary the lattice spacing $a$ (by changing $\beta$ ) and tune the bare parameters $\kappa$ and $\lambda$ to keep two dimensionless physical quantities $F_{1}$ and $F_{2}$ constant. This procedure corresponds to the renormalisation of $\kappa$ and $\lambda$ and defines in the parameter space a so called line of constant physics. A sensible choice would be to find $F_{1}$ strongly dependent on $\kappa$ and $F_{2}$ strongly dependent on $\lambda$.

We consider the quantity $F_{1}=r_{0}\left[2 \mu-V_{0}\left(r_{0}\right)\right]$ : it mainly depends on the value of the mass of the dynamical Higgs field which is in turn determined by the choice of $\kappa$. At $\beta=2.4$ we find

$F_{1}=r_{0}\left[2 \mu-V_{0}\left(r_{0}\right)\right]=F_{1}^{*} \equiv 1.26$.

For different values of $\lambda$, we match the parameter $\kappa$ at $\beta=2.2$ using the condition eq. (田). The results are shown in Fig. 3. As a by-product of these simulations we obtain that

$\frac{a(\beta=2.2)}{a(\beta=2.4)} \approx 1.9$.

We studied several candidates 顿 for a quantity $F_{2}$ which is sensitive to a variation of the parameter $\lambda$ once the parameter $\kappa$ is matched using the 


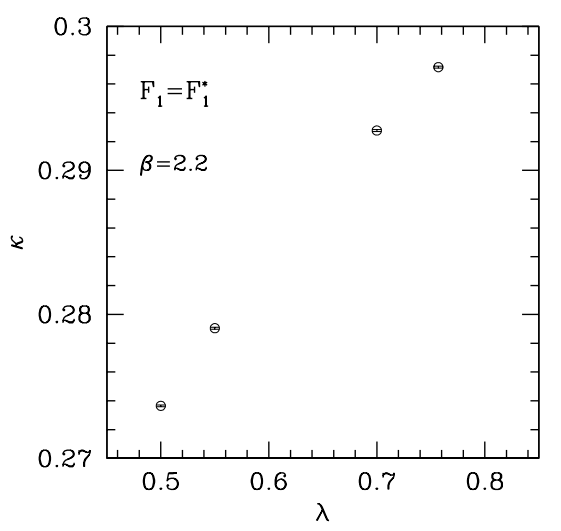

Figure 3. Here, we show the matching of $\kappa$ at $\beta=2.2$ using the matching condition eq. (4).

condition eq. (4). We cannot draw a definitive conclusion yet. Our results support earlier observations [7] that the physics in the confinement "phase" of the SU(2) Higgs model is weakly dependent on the parameter $\lambda$. We use this as an assumption and study the scaling behavior of the static potentials between $\beta=2.2$ and $\beta=2.4$ by using only the matching condition for $F_{1}$ eq. (位).

In Fig. 4, we compare the results for the ground state and first excited state static potentials. They show compatibility with scaling within minute errors! We have also computed the static potentials at $\beta=2.2$ for other values of $\lambda$ in the range $0.5 \leq \lambda<0.8$. No dependence on $\lambda$ is seen. These results are a strong indication for a continuum-like behavior of the static potentials already at the small $\beta$ values that we use.

\section{Conclusions}

We clearly observe string breaking as level crossing phenomenon in the confinement "phase" of the $\mathrm{SU}(2)$ Higgs model. The static potentials show a remarkable scaling behavior under variation of the lattice spacing by almost a factor two. The search for a method of defining lines of constant physics in the confinement "phase" of the SU(2) Higgs model has only partially been solved (Fig. 3). Finding a renormalised observable sensi-

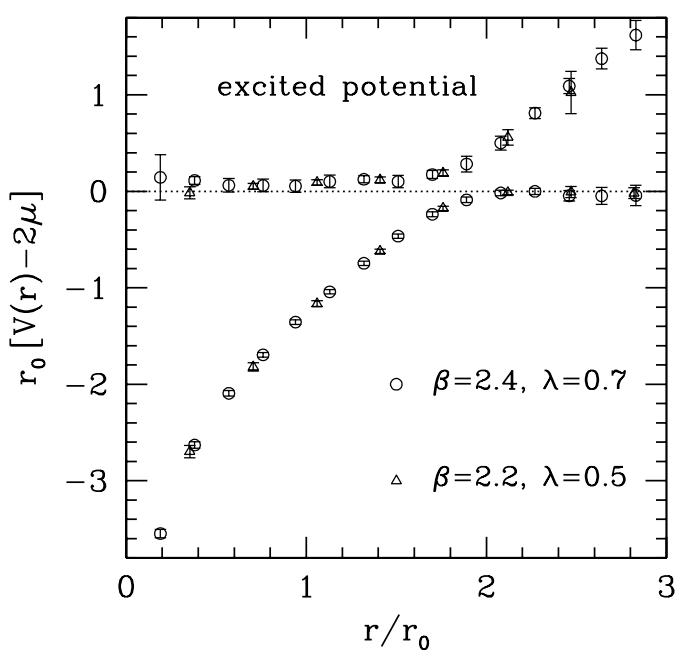

Figure 4. Here, we show the scaling of the renormalised ground state and first excited state static potentials. The value of $\kappa$ is matched to keep $F_{1}=F_{1}^{*}$.

tive to $\lambda$ is still an open and interesting question.

I thank R. Sommer for his constant support and the Konrad-Zuse-Zentrum für Informationstechnik Berlin (ZIB) for granting CPU-resources to this project.

\section{REFERENCES}

1. For a review see K. Schilling, these proceedings.

2. C. Michael, Nucl. Phys. B (Proc. Suppl.) 26 (1992) 417.

3. ALPHA, F. Knechtli and R. Sommer, Phys. Lett. B440 (1998) 345, erratum: Phys. Lett. B454 (1999) 399.

4. F. Knechtli, Ph.D. thesis, to appear in heplat.

5. M. Lüscher and U. Wolff, Nucl. Phys. B339 (1990) 222.

6. R. Sommer, Nucl. Phys. B411 (1994) 839.

7. I. Montvay, Nucl. Phys. B269 (1986) 170.

8. I.T. Drummond, Phys. Lett. B434 (1998) 92. 